\title{
Diagnostic systems for the nuclear fusion and plasma research in the PF-24 plasma focus laboratory at the IFJ PAN
}

Łukasz Marciniak, Anna Wójcik-Gargula, Agnieszka Kulińska, Jakub Bielecki, Urszula Wiącek

\begin{abstract}
This paper presents a set of diagnostics dedicated to PF-24 - new medium size - plasma focus (PF) device built and operated at the Institute of Nuclear Physics Polish Academy of Sciences (IFJ PAN). The PF-24 can operate at energy level up to $93 \mathrm{~kJ}$ and charging voltage up to $40 \mathrm{kV}$. Each condenser is connected with a specially designed spark gap with a very small jitter, which ensures a high efficiency and a low current rise time. The working parameters of PF-24 generator make it a suitable tool for testing new detection systems to be used in fusion research. Four types of such detection systems are presented in this article: three diagnostic systems used to measure electric quantities (Rogowski coil, magnetic probe, capacitance probe), neutron counter based on beryllium activation, fast neutron pinhole camera based on small-area BCF-12 plastic scintillation detectors and high-speed four-frame soft X-ray camera with microchannel plate.
\end{abstract}

Key words: diagnostics for DD fusion products $\bullet$ fast neutron sources $\bullet$ plasma diagnostics $\bullet$ plasma focus $\bullet$ plasma imaging

Ł. Marciniak ${ }^{\bowtie}$, A. Wójcik-Gargula, A. Kulińska,

J. Bielecki, U. Wiącek

Department of Radiation Transport Physics,

Institute of Nuclear Physics Polish Academy of Sciences,

152 Radzikowskiego Str., 31-342 Kraków, Poland,

Tel.: +48 12662 8167, Fax: +48 12662 8335,

E-mail: lukasz.marciniak@ifj.edu.pl

Received: 13 May 2016

Accepted: 30 May 2016

\section{Introduction}

Plasma focus (PF) device belongs to the family of dynamic non-cylindrical Z-pinches. In this device hot dense magnetized plasma is created as a result of a discharge between coaxial electrodes over a surface of isolator in a working gas. If the working gas is deuterium, then DD fusion reactions take place, in the so-called plasma column or plasma pinch, which is created at the end of a central electrode (anode) [1-4].

Over the last few years, the interest in plasma focus devices has grown because they are efficient sources of fusion neutrons, fast ions, soft and hard $\mathrm{X}$-rays, and at the same time, they are relatively cheap in exploitation. Thus, they can be used in

- basic research on plasma physics and nuclear fusion,

- testing new plasma and nuclear fusion detectors and diagnostic systems,

- study on the mechanism of nuclear fusion reactions, and

- study of plasma-wall interactions.

Moreover, the optimization of the operation of PFs can help to obtain more efficient and intense sources of neutron and X-ray radiation, which can find use in different branches of science.

The discharge in the Mather-type PF devices can be divided into three main stages: breakdown and 
current sheath build-up phase, run-down (or acceleration) phase, and radial compression (or pinch) phase. In most types of PF devices, the duration of the first and the second phase is in the order of few microseconds. The third phase usually lasts up to a few hundreds of nanoseconds. Moreover, the plasma column can have a length from a few millimeters to dozens millimeters [5-8]. In order to study such short-lived phenomena and plasma objects with rather small size, proper diagnostic systems are required.

The main goal of the experiments carried with the use of diagnostic systems presented in this article is further studies of plasma physics and nuclear fusion in the PF devices. This refers mainly to studying the role of plasma outflow, role of dissipation of the magnetic field into plasma pinch, nature of neutron emission and defining the properties of the plasma structure at the final stage of pinch. In general, there are still many unknowns in physical processes that take place in Z-pinch systems. Experimenters trying to explain them stated angular anisotropy of X-ray emission during different stages of pinch from different areas. This happens for soft X-ray and hard X-ray - two mechanisms of emission are simultaneously active. Moreover, it is often suggested that majority of X-ray energies emitted from pinch are higher than $50 \mathrm{keV}$ and are emitted during last stage of pinch (often second neutron pulse) from so-called hot spots. Also increase in yield of neutrons in the last stage of pinch, angular anisotropy in neutron emission (polar anisotropy between the side-on and end-on directions) from the plasma and observation of the Doppler shift in the neutron energy at backward angels, were registered. This all suggests that the nature of neutron emission is rather based upon the occurrence of suprathermal deuterons and the beam-target mechanism with accompanying emission of hard X-rays. This question is closely related to the plasma pinch symmetry, plasma dynamics, and physics of conversion of a magnetic field to the pinch plasma.

\section{The apparatus}

PF-24 - the new plasma focus device of a Mather-type built at the Institute of Nuclear Physics Polish Academy of Sciences (IFJ PAN) - can operate at the energy level up to $93 \mathrm{~kJ}$. The condenser bank of PF-24 consists of 24 low-inductance condensers connected in parallel and grouped in three modules, eight condensers each. The total capacitance of the system is about $116 \mu \mathrm{F}$. The charging voltage can be adjusted between 16 and $40 \mathrm{kV}$. The specially designed spark gaps with a very small jitter ensures a short current rise time of about $1.6 \mu$ s and high efficiency. Hence, the plasma produced and sustained at the pinch phase of the plasma focus discharge can be used as an intense source of radiation. The yield of neutrons obtained with PF-24 is usually in a range of $10^{8}$ to $10^{10}$ neutron per discharge when operating with deuterium as a working gas at a pressure of 2 mbar and a battery charging voltage of $16 \mathrm{kV}$. The

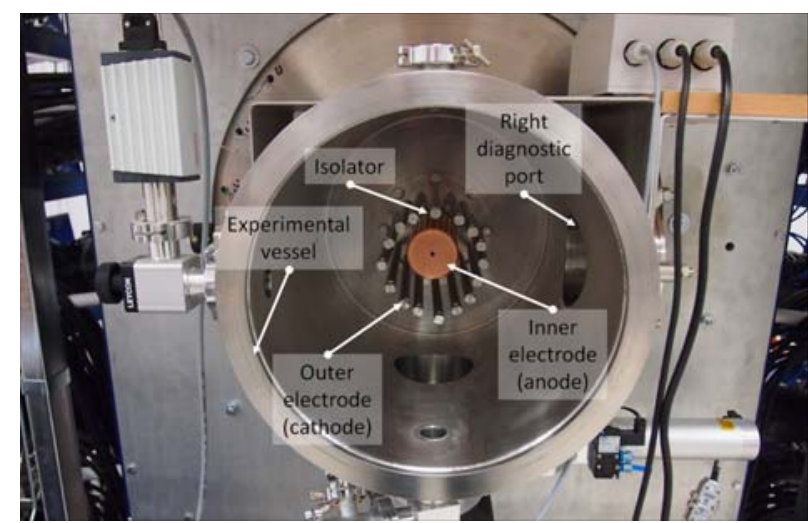

Fig. 1. The view of the open PF-24 experimental chamber.

electrode structure of the PF-24 device consists of two coaxial electrodes: center and outer electrodes of 62 and $110 \mathrm{~mm}$ in diameter, respectively. The central electrode is made of one solid oxygen-free copper rod while the outer electrode consists of 16 stainless steel rods [9]. The front view of the open experimental chamber of the PF-24 device is shown in Fig. 1.

The abovementioned specification of PF-24 device and the various test experiments favour this plasma focus as a good instrument for testing new diagnostics dedicated to nuclear fusion and plasma research.

\section{Diagnostics dedicated to the PF-24 device}

Four different types of diagnostic systems dedicated to the PF-24 are presented below. These diagnostic systems include: the sets of diagnostics for the measurement of electric quantities, neutron activation counter, fast neutron pinhole camera based on small-area BCF-12 plastic scintillation detectors and high-speed four-frame soft X-ray camera with a microchannel plate.

\section{Measurements of electric quantities}

In order to measure electric quantities, during the different stages of a discharge in the PF-24 device, the Rogowski coil, magnetic probe, and capacitance probe are used as the standard diagnostics. They provide the information on the overall time of plasma sheath acceleration and compression, as well as on the character of compression and plasma pinch. It means also that one can investigate how the power delivered to the system changes with time during a discharge and how electrode size influence the radiation emission yield [10].

The Rogowski coil is used to measure the current flowing into the collector area. Its calibration factor was determined to be $26.4 \mathrm{kA} / \mathrm{V}$. The measurements with the magnetic probe give the information about the time derivative of current and thus help to determine the character of compression of current sheath and the rise time of current as well as to control a homogenous current flow around the collector. The capacitance probe is used to define the voltage 


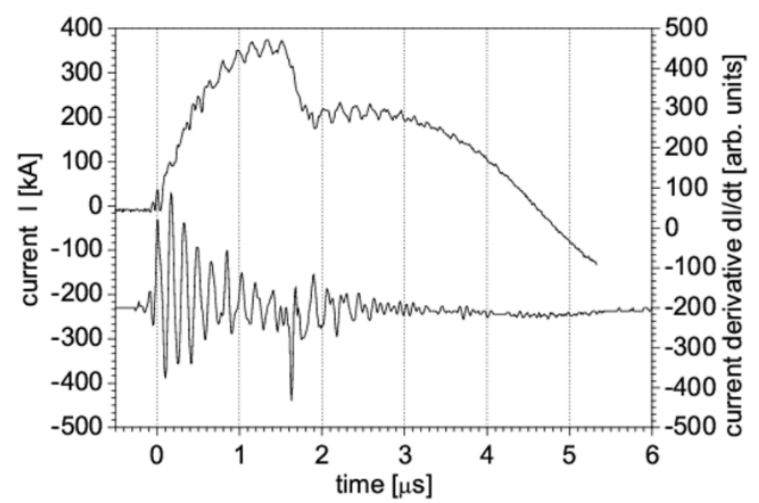

Fig. 2. The waveform of current $I$ (top graph), and the time derivative of current $\mathrm{d} I / \mathrm{d} t$ (bottom graph) of the PF-24 discharge \#16022407 $\left(U_{0}=16 \mathrm{kV}, p_{0}=1.50 \mathrm{mbar}\right.$, working gas $\mathrm{D}_{2}, Y_{\mathrm{n}}=1.75 \times 10^{8} \mathrm{n} /$ dis $)$.

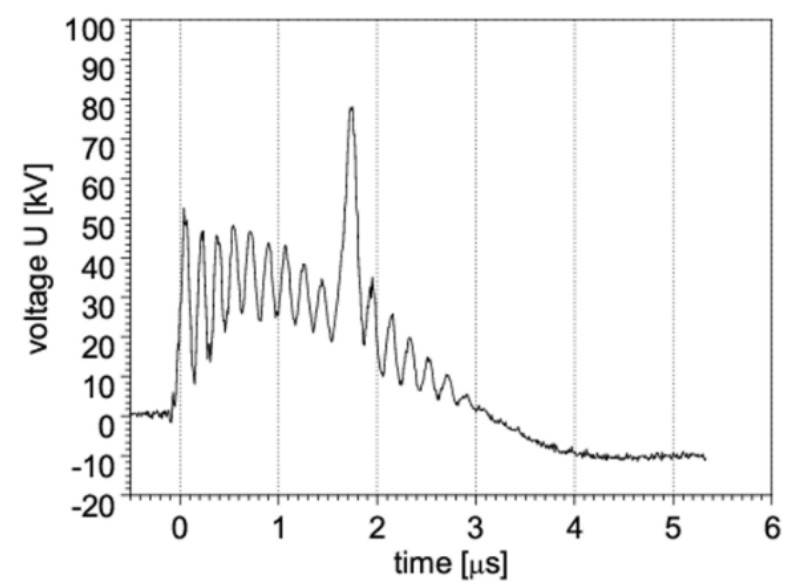

Fig. 3. The waveform of voltage $(U)$ of the PF-24 discharge \#16022407 $\left(U_{0}=16 \mathrm{kV}, p_{0}=1.50\right.$ mbar, working gas $\mathrm{D}_{2}$, $Y_{\mathrm{n}}=1.75 \times 10^{8} \mathrm{n} /$ dis $)$.

induced during the maximal compression phase. Hence, it gives the information about the quality of compression. The calibration factor of the capacitive voltage probe in the PF-24 is equal to $3.77 \mathrm{kV} / \mathrm{V}$ for the pulse lasted less than $1.5 \mathrm{~ms}$ [9].

Figures 2 and 3 present the current, the time derivative of current and the voltage of the PF-24 discharge \#16022407 under 1.5 mbar pressure of $\mathrm{D}_{2}$ and charging voltage of $16 \mathrm{kV}$. The total neutron yield (measured as described in the next section) for this discharge is about $1.75 \times 10^{8} \mathrm{n} /$ dis.

\section{Neutron counter based on beryllium activation}

Due to the intense fast neutron emission from the PF devices in a short timescale, the measurements of total yield of fusion neutrons during a single discharge is a challenging task. Therefore, to estimate the total yield of fusion neutrons during a single discharge, a neutron activation counter consisting of a beryllium plate $(100 \mathrm{~mm} \times 100 \mathrm{~mm}, 2 \mathrm{~mm}$ thick $)$ was designed. Its goal is to register characteristic $2.45 \mathrm{MeV}$ neutrons from DD fusion reaction. A large gas area of SP-126C proportional counter, produced by Canberra, serves as a detector for electrons emitted from ${ }^{6} \mathrm{He}$ nuclei. The calibration coefficient, determined based on the results of Monte Carlo

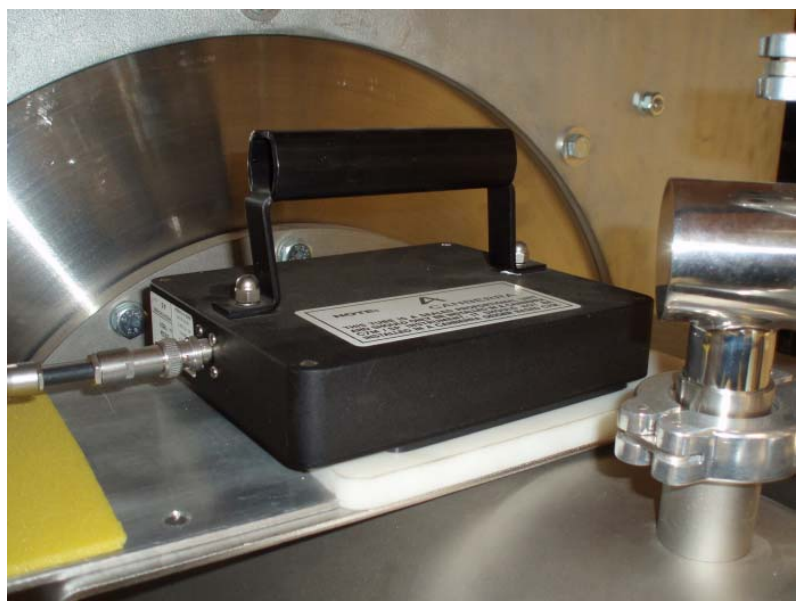

Fig. 4. Beryllium neutron activation counter dedicated to the PF-24 device.

simulations using the MCNP code [11] and the measurements with PuBe source, is equal to about $2 \times 10^{5}$. On average, the total neutron yields from the PF-24 device registered with this counter ranges from about $10^{8}$ to about $10^{10} \mathrm{n} /$ dis [9] .

In Fig. 4, the actual view of beryllium neutron activation counter placed above the PF-24 experimental chamber is presented.

\section{Fast neutron pinhole camera based on small-area} BCF-I 2 plastic scintillation detectors

The small-area scintillation detectors were designed and constructed at the IFJ PAN to work as a part of a detection system for a neutron pinhole camera [12]. In Fig. 5, the actual view of the neutron pinhole camera placed on the mobile rack is presented (rear view of the camera without the detection part).

Due to the specific nature of neutron interactions with matter, the shielding and collimators of the neutron pinhole camera were optimized by means of Monte Carlo simulations using the MCNP code

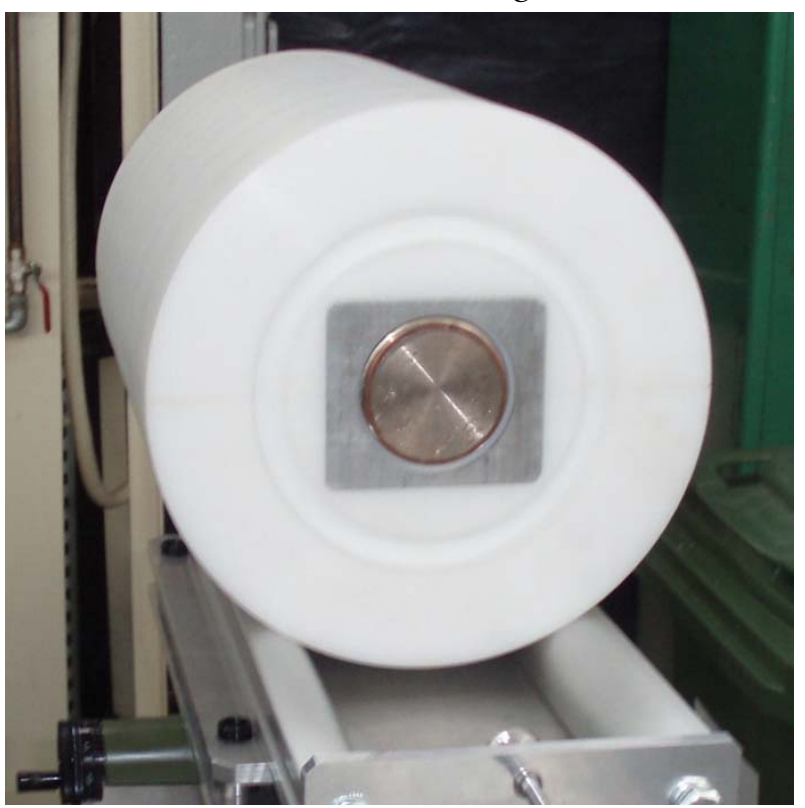

Fig. 5. Neutron pinhole camera developed and constructed at the IFJ PAN. 
calculations. The final calculations were made for yield $2 \times 10^{8}$ neutrons per discharge and for minimal fluence of 100 neutrons per square $\mathrm{cm}$ of a single detector. The size of pinhole was chosen as $0.17 \mathrm{~cm}$. The total length of camera body is $580 \mathrm{~mm}$, while the distance from the front hole to pinhole and the distance from pinhole to linear matrix of detectors is 46 and $316 \mathrm{~mm}$, respectively. The camera inner-walls, which constitute the collimator were made from copper (two anti-scattering cones are applied) and cadmium because they are very good absorbers of neutrons. In turn, the outer shielding was made from polyethylene, which aims at reducing influence from already scattered neutrons into different angels. More details on the pinhole camera design can be found in Ref. [12].

The detection system of the neutron pinhole camera consists of four small-area BCF-12 plastic scintillation detectors arranged in a linear array. This is due to the need of neutron registration with spatial resolution. The detectors' scintillation material is BCF-12 plastic scintillator (Saint Gobain) in a form of $5 \mathrm{~mm} \times 5 \mathrm{~mm} \times 60 \mathrm{~mm}$ rod. The material emits blue light, which is transferred via $25 \mathrm{~m}$ long optical fiber (Mitsubishi) to a photomultiplier tube (H3164-10, Hamamatsu). BCF-12 scintillation detectors enable the measurement of $2.45 \mathrm{MeV}$ neutrons and X-ray radiation with a nanosecond time resolution and relatively high efficiency [12].

Figure 6 shows the waveform registered with the small-area BCF-12 plastic scintillation detector during the discharge \#14101005 in the PF-24 device. Since the detector was placed at the distance of about $2 \mathrm{~m}$ from the experimental chamber (and not in the pinhole camera body), the pulses from neutrons and hard X-ray (HXR) radiation can be easily distinguished. The soft X-ray radiation signal is not seen because of absorption in the experimental chamber walls. Additionally, a $1 \mathrm{~cm}$ thick Bi plate was set in the front of the detector to attenuate the HXR signal.

\section{High speed four-frame soft X-ray camera with microchannel plate}

The high speed four-frame soft X-ray camera (HS-4F-SXRC) was designed and constructed by ACS Laboratory (Warsaw, Poland) for imaging of the plasma column of PF-24 in extreme ultraviolet

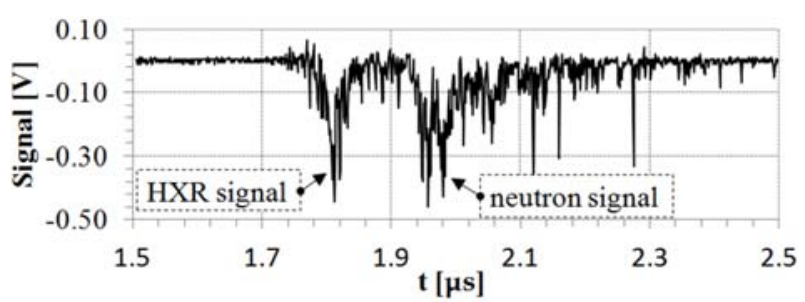

Fig. 6. Waveform registered, with the use of the small-area BCF-12 plastic scintillation detector, for the discharge \#14101005 in the PF-24 device. Hard X-ray (HXR) and neutron signals are marked. The source-to-detector distance was set to $2 \mathrm{~m}$. A $1 \mathrm{~cm}$ thick Bi plate was placed in the front of the detector. and soft X-ray spectral ranges (energy band between about $8 \mathrm{eV}$ to $6 \mathrm{keV}$ ). In general, from the properly recorded 2D images of plasma X-ray emissivity, the distribution of electron concentration, temperature, and geometry of plasma column can be obtained.

In general, the HS-4F-SXRC camera system consists of few main elements: a vacuum track, a pumping systems, a diaphragm, a microchannel plate (MCP), a CCD camera with appropriate optics and other electronic devices.

The vacuum track is divided into two parts, which have separated vacuum systems. The first part is connected directly to the right diagnostic port of the PF-24 experimental chamber and ends with the diaphragm. The diaphragm is the element with four cone-shaped pinholes than can be covered by polystyrene, beryllium, or aluminium blocking foils, which serve as radiation filters but also divide the vacuum track into two parts. This helps to prevent the change of pressure and deuterium outflow from the experimental chamber. Also just before pinholes three plastic plates are placed with four holes tailored in such a way to extinguish plasma shock waves.

The second part of the vacuum track starts with diaphragm and ends with MCP. The MCP constitutes the basic registration system, amplifier, and converter of EM radiation into electron current and then to visible light. At the end of it, an image of plasma column cross section is created. The image is generally inverted and enlarged and then sent to the IT CCD array camera through appropriate optics. The MCP's round layers are divided electrically into four equal triangular measuring sections, which correspond to the position of the pinholes. Each frame of the HS-4F-SXRC camera represents one section. The pinholes differ only with a very small angle so all of them 'observe' the image from the same line of sight but during different time intervals. This is due to the gating pulses that can be properly set. In general, each sector of the MCP can be gated by only one positive electrical impulse, which is applied between a phosphor screen and an input side of the MCP (connected to the common ground). The often problem is when the first triggering impulse should be send to activate the first sector/frame (position in time of the pinch phenomena can change). In other words, additional registration system somehow coupled with the beginning of the compression phase is required. This sometimes is achieved through laser systems or can be achieved through putting proper fiber into the experimental chamber.

In the CCD camera, the signal from the four frames is digitalized and acquired in on-board mounted memory. All of the frames are saved into one image with dimensions $44 \mathrm{~mm} \times 44 \mathrm{~mm}$. The limiting image spatial resolution (in reference to central PF-24 device plane perpendicular to line-of-sight) is 0.5 line pairs per millimeter $(\mathrm{LP} / \mathrm{mm})$. For the HS-4F-SXRC dedicated to the PF-24 device, the distance from a plasma column to MCP, the distance from the plasma column to the pinholes and the distance from pinholes to the MCP is about 800 , 550 , and $250 \mathrm{~mm}$, respectively. This gives a reduc- 


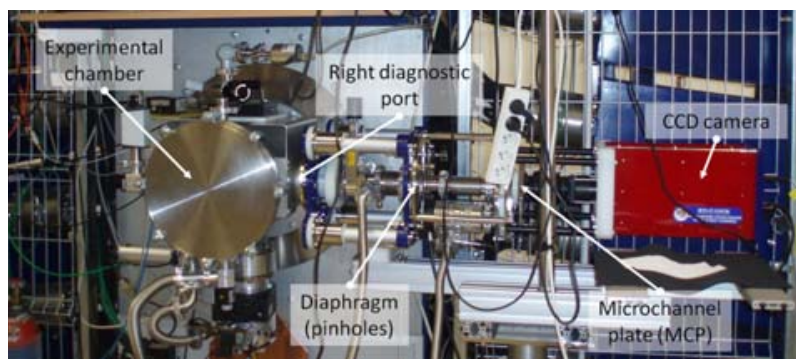

Fig. 7. High-speed four-frame soft X-ray camera with a microchannel plate connected to the PF-24 device.

tion of the image size but the CCD camera optics enlarges the image - the magnification factor is equal to about 2.2. More details on the experiments with the use of HS-4F-SXRC system can be found in Ref. [13]. In Fig. 7, the actual view of the HS-4F-SXRC camera connected to the PF-24 device experimental chamber is shown.

Figure 8 shows one of the first images of the evolution of the plasma column obtained during the discharge \#15090917 in the PF-24 device. The colour map represents the plasma emissivity of the soft X-rays given in arbitrary units.

The four frames in Fig. 8 were captured at different time intervals, one after the other, starting from the left frame and then clockwise. Also the diameters of pinholes corresponding to the frames are decreasing clockwise starting from the left frame. This means the lower diameter of the pinholes were for this image: 97, 106, 125, and $140 \mu \mathrm{m}$. The interval between the first and the second frame as well as the interval between the third and the fourth frame was $16.5 \mathrm{~ns}$. On the other hand, the interval between second and third frame was $20.0 \mathrm{~ns}$. The registration time of each frame was no longer than 1.8 ns. Furthermore, up till now, the position in time of each frame in reference to the moment of the maximal compression level was not established. Also, no blocking foils were used during the time of gathering of these data.

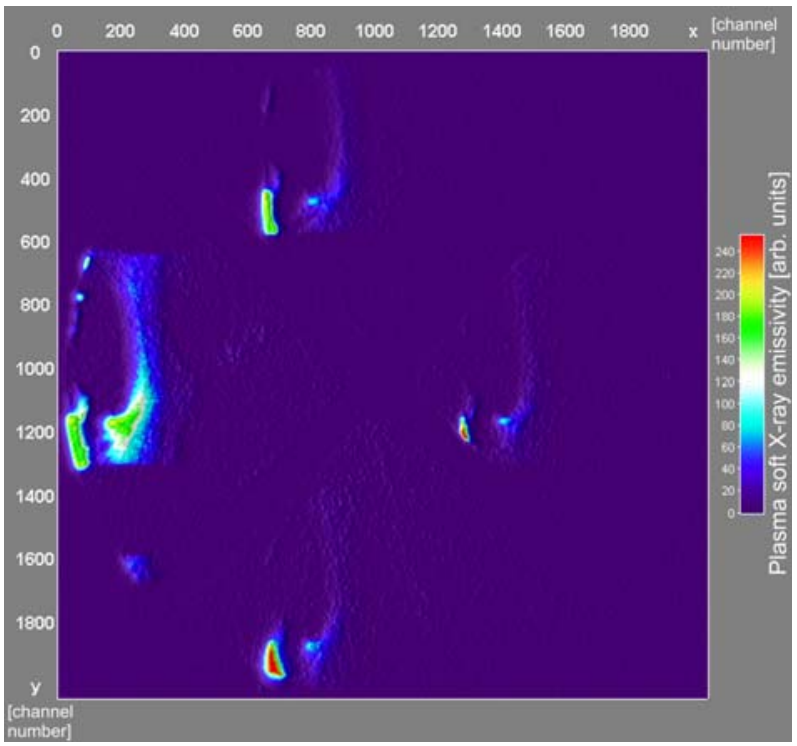

Fig. 8. Evolution of the plasma column in the PF-24 device - in the discharge \#15090917. The left frame is the first one in timescale and the rest goes clockwise.
The $0 \mathrm{X}$ and $0 \mathrm{Y}$ axes in Fig. 8 present the number of channel - from 0 to 2048, although, the actual size of network is only $512 \times 512$ elements because it is the maximal resolution in the Image Interactive 3D Surface Plot (data recalculated into bigger elements automatically). The data on the $0 \mathrm{Z}$ axis are presented as 'Thermal LUT' from 0 to 256 - the red colour indicates higher plasma soft X-ray emissivity (data without additional smoothing). The 'cutoffs' of plasma column in the picture, best visible in the first and the second frame, are caused by the stainless steel cathode rods which unfortunately partially shielded the detector from radiation.

\section{Conclusion}

Four different types of diagnostic systems have been described in this article. They are designed to measure radiation emitted from hot dense magnetized plasma as well as to study some basic electric parameters. They provide information on current, time derivative of current and voltage in the collector area, total neutron yield, neutron and X-ray signals, and plasma X-ray emissivity. Together they supply a good quality of registered information.

As it has been pointed in the introduction section, there are a variety of effects that should be studied in the PF devices. This is why different diagnostic techniques should be used. Because, for example, through analysis of the waveforms acquired from electric diagnostic systems phases of discharge can be distinguished (the moment of beginning and the moment of end of each phase in timescale). This includes determination of some characteristic points of the plots such as value of the maximal current. This information is needed for the evaluation of the discharge - how close to anticipated perfect current, voltage, and current derivative waveform shape it was. This also enables to choose which shots should be chosen for statistical calculations and assumptions as well as for comparison with the simulated data from different theoretical models. Moreover, the waveforms can be used for making approximated calculations of physical parameters for each phase of discharge i.e., value of current sheath velocity.

The total neutron yield helps to give information about character of compression - how strong or efficient it was. It is also used when assumptions about plasma focus scaling laws are made as well as when assessment of simulation and theoretical model is made.

Registered signals of neutrons and X-rays give detailed information about intensity of radiation changes in timescale thus they can help to understand the phenomena happening during pinch evolution.

The plasma X-ray emissivity gives direct information about pinch evolution in time and space. These data are most helpful when distinguishing objects in plasma or regions of interest in pinch evolution. They also enable acquiring some direct information for comparison with data acquired from models i.e., effective radius of plasma column. 
In conclusion, further studies with the use of all these diagnostic tools may provide new insights on fusion reaction mechanisms in plasma focus devices.

Although the presented diagnostic systems are designed for the PF devices, they use technical solutions and methods that can find the applications in research with the use of other fusion devices.

Acknowledgments. The scientific research of the corresponding author of this article is supported by Marian Smoluchowski Krakow Research Consortium "Matter-Energy-Future" from the grant KNOW. The authors would like to thank Krzysztof Tomaszewski and the entire team of the ACS Laboratory for making available the 4F-HS-SXRC diagnostic equipment and for participation in the first experiments with this diagnostic in the PF-24 plasma focus laboratory.

\section{References}

1. Petrov, P. P., Filippov, N. V., Filippova, T. I., \& Khrabrov, V. A. (1960). Powerful pulsed gas discharges in chambers with conducting walls. In M. A. Leontovich (Ed.), Plasma physics and the problem of controlled thermonuclear reactions (Vol. 4, pp. 198-212). New York: Pergamon Press.

2. Filippov, N. V., Filippova, T. I., \& Vingredov, V. P. (1962). Dense, high-temperature plasma in a noncylindrical Z-pinch compression. Nucl. Fusion Suppl., 2, 577-587.

3. Mather, J. W. (1964). Investigation of the high-energy acceleration mode in the coaxial gun. Phys. Fluids, 7(11), S28-S34. DOI: 7/11/10.1063/1.1711086.

4. Mather, J. W. (1965). Formation of a high-density deuterium plasma focus. Phys. Fluids, 8(2), 366-377. DOI: 8/2/10.1063/1.1761231.

5. Herold, H., Jerzykiewicz, A., Sadowski, M., \& Schmidt, A. (1989). Comparative analysis of large plasma focus experiments performed at IPF. Stuttgart, and IPJ, Świerk. Nucl. Fusion, 29(8), 1255-1266. DOI: $10.1088 / 0029-5515 / 29 / 8 / 002$.

6. Bernard, A., Bruzzone, H., Choi, P., Chuaqui, H., Gribkov, V., Herrera, H., Hirano, K., Krejci, A., Lee, S., Luo, C., Mezzetti, F., Sadowski, M., Schmidt, H., Ware, K., Wong, C. S., \& Zoita, V. (1998). Scientific status of plasma focus research. J. Moscow Phys. Soc., 8, 93-170.

7. Scholz, M., Karpinski, L., Krauz, V. I., Kubes, P., Paduch, M., \& Sadowski, M. J. (2012). Progress in MJ plasma focus research at IPPLM. Nukleonika, 57(2), 183-188.

8. Krauz, V., Mitrofanov, K., Myalton, V., Koidan, V., Mokeev, A., Vinogradov, V., Vinogradova, Yu., Scholz, M., Paduch, M., Karpinski, L., Zielinska, E., \& Kubes, P. (2013). Recent results of studies of magnetic field distribution and neutron scaling on PF-1000 and PF-3 facilities. Probl. At. Sci. Tech., 83, 114-118.

9. Scholz, M., Marciniak, L., Kulinska, A., Karpinski, L., Igielski, A., Wojcik-Gargula, A., Bielecki, J., Krol, K., \& Drozdowicz, K. (2016). Initial results from PF-24 - a 100 kJ Plasma Focus device. IEEE Trans. Plasma Sci. (submitted).

10. Scholz, M. (2014). Plasma-focus and controlled nuclear fusion. Habilitation dissertation. Kraków: Institute of Nuclear Physics PAN.

11. Bieńkowska, B., Prokopowicz, R., Scholz, M., Kaczmarczyk, J., Igielski, A., Karpinski, L., Paduch, M., \& Pytel, K. (2014). Neutron counter based on beryllium activation. AIP Conf. Proc., 1612, 105-108.

12. Bielecki, J., Wojcik-Gargula, A., Wiacek, U., Scholz, M., Igielski, A., Drozdowicz, K., \& Woznicka, U. (2015). A neutron pinhole camera for PF-24 source: Conceptual design and optimization. Eur. Phys. J. Plus, 130(7), 145. DOI: 10.1140/epjp/i2015-15145-x

13. Kasperczuk, A., Paduch, M., Tomaszewski, K., Zielinska, E., Miklaszewski, R., \& Szymaszek, A. (2016). A plasma focus device as a metallic plasma jet generator. Laser Part. Beams, 34(2), 356-361. DOI: 10.1017/ S0263034616000215. 